T. Shimomura

Nagoya Math. J.

Vol. 104 (1986), 117-127

\title{
TOPOLOGICAL ENTROPY AND PERIODIC POINTS OF A FACTOR OF A SUBSHIFT OF FINITE TYPE
}

\author{
TAKASHI SHIMOMURA
}

\section{§. Introduction}

Let $X$ be a compact space and $f$ be a continuous map from $X$ into itself. The topological entropy of $f, h(f)$, was defined by Adler, Konheim and McAndrew [1]. After that Bowen [4] defined the topological entropy for uniformly continuous maps of metric spaces, and proved that the two entropies coincide when the spaces are compact. The definition of Bowen is useful in calculating entropy of continuous maps.

By improving on the definition of entropy given in [1] and [4], we have the following results.

Theorem 1. Let $f: X \rightarrow X$ be a continuous map on a compact space $X$ and let $g: Y \rightarrow Y$ be a continuous map on a compact Hausdorff space $Y$. Suppose there is a continuous map $\pi: X \rightarrow Y$ such that $\pi(X)=Y$ and $g \circ \pi$ $=\pi \circ f$. Then

$$
h(f) \leqq h(g)+\sup _{y \in Y} h\left(f, \pi^{-1}(y)\right) .
$$

This is a generalization of the result of Bowen (Theorem 1.7, [4]). As a corollary of Theorem 1 we have

Corollary 2. Let $E, X$ and $G$ be compact Hausdorff spaces. Suppose that $\pi: E \rightarrow X$ is a projection of a fiber bundle with the total space $E$, the base space $X$ and the structure group $G$. If $f: E \rightarrow E$ is a bundle map and $f^{\prime}: X \rightarrow X$ is its base map, then $h(f)=h\left(f^{\prime}\right)$ holds.

Introducing a new method of calculating entropy, we have

THEOREM 3. Let $\sigma: \Sigma \rightarrow \Sigma$ be a topologically mixing subshift of finite type and let $f: X \rightarrow X$ be a continuous map on a compact metric space $X$. Suppose there exists a continuous map $\pi: \Sigma \rightarrow X$ such that $\pi(\Sigma)=X$ and

Received April 10, 1985. 
$f \circ \pi=\pi \circ \sigma . \quad$ Then

$$
h(f) \leqq \liminf _{n \rightarrow \infty}(1 / n) \log N_{n}(f),
$$

where $N_{n}(f)$ is the cardinal number of the set $\left\{x \in X ; f^{n}(x)=x\right\}$ and $\log N_{n}(f)=\infty$ if $N_{n}(f)$ is not finite.

As a corollary of Theorem 3 , we can give a partial answer for a problem stated in Walters (p. 180, [8]). More precisely

Corollary 4. Let $X$ be a compact metric space and $f: X \rightarrow X$ be an expansive homeomorphism. If $(X, f)$ is a factor of a topologically mixing subshift of finite type, then

$$
h(f)=\lim _{n \rightarrow \infty}(1 / n) \log N_{n}(f) .
$$

Bowen proved in $((2.8),[2])$ that if $f$ is expansive then

$$
h(f) \geqq \limsup _{n \rightarrow \infty}(1 / n) \log N_{n}(f) .
$$

From this result together with Theorem 4, Corollary 4 is obtained.

The author would like to thank Prof. N. Aoki and Prof. K. Shiraiwa for encouragement and effort to form the paper.

\section{$\S 1$. Definitions and basic properties}

Hereafter $X$ is a compact space and $f$ is a continuous map of $X$ into itself. By $(X, f)$ we denote the dynamical system of $X$ and $f$.

Let $\alpha$ be a finite open covering of $X$ and ${ }^{*} \alpha$ denote the cardinality of $\alpha$. For $K$ a subset, we put

$$
N_{K}(\alpha)=\min \left\{\sharp \beta: \beta \subset \alpha, K \subset \bigcup_{B \in, 3} B\right\}
$$

and for $n>0$

$$
\alpha_{f}^{n}=\left\{\bigcap_{i=0}^{n-1} f^{-i}\left(A_{i}\right): A_{i} \in \alpha, 0 \leqq i \leqq n-1\right\} .
$$

Further we define

$$
\begin{gathered}
h(f, K, \alpha)=\limsup _{n \rightarrow \infty}(1 / n) \log N_{K}\left(\alpha_{f}^{n}\right), \\
h(f, K)=\sup _{\alpha} h(f, K, \alpha)
\end{gathered}
$$

where the supremum is taken for all the finite open covering of $X$. If in particular $K=X$, then we write $h(f)=h(f, K)$. This is in the case given in [1]. 
Remark 1. If $K$ is closed and $f(K) \subset K$, then the existence of

$$
\lim _{n \rightarrow \infty}(1 / n) \log N_{K}\left(\alpha_{i}^{n}\right)
$$

is easily checked (cf. see [1]).

Remark 2. If $(X, d)$ is the metric space and $K$ is closed, then $h(f, K)$ coincides with entropy given in [4].

In the rest of this section we investigate the properties of the definition of entropy given here.

Let $\alpha=\left\{A_{1}, \cdots, A_{t}\right\}$ be a finite family of closed sets of $X$ and denote by $M=\left(M_{i j}\right)$ a $t \times t$-matrix of 0 's and 1's. We say that a pair $(\alpha, M)$ is a CM-pair for $f$ if for any $x \in X$ there is a sequence

$$
x^{*}=\left(x_{0}, x_{1}, \cdots\right) \in \prod_{i=0}^{\infty} S(i) \quad(S(i)=\{1, \cdots, t\}, i \geqq 0)
$$

such that $M_{x_{i} x_{i+1}}=1(i \geqq 0)$ and $x \in \bigcap_{i=0}^{\infty} f^{-i}\left(A_{x_{i}}\right)$.

Remark 3. Under the notations, let us put

$$
\Sigma=\left\{x=\left(x_{0}, x_{1}, \cdots\right) \in \prod_{0}^{\infty} S(i): M_{x_{i} x_{i+1}}=1(i \geqq 0)\right\} .
$$

Then $\Sigma$ is closed. We define a shift $\sigma$ usually by

$$
\sigma(x)_{i}=x_{i+1} \quad(i \geqq 0) .
$$

Obviously $\sigma(\Sigma)=\Sigma$. Such a shift $\sigma: \Sigma \rightarrow \Sigma$ is called to be a one side subshift of finite type.

Fix $n \geqq 1$. A finite sequence $\left(x_{0}, x_{1}, \cdots, x_{n-1}\right)$ is said to be an admissible sequence of length $n$ if $M_{x_{i} x_{i+1}}=1$ for $0 \leqq i \leqq n-1$. Let $E_{n}$ denote a set of admissible sequences of length $n$. We say that $E_{n}$ is separated if for any distinct points $\left(x_{0}, \cdots, x_{n-1}\right),\left(y_{0}, \cdots, y_{n-1}\right) \in E_{n}$ there is $0 \leqq j \leqq$ $n-1$ such that $A_{x_{j}} \cap A_{y_{j}}=\phi$. For $K$ a subset we say that an admissible sequence $\left(x_{0}, \cdots, x_{n-1}\right)$ is attached to $K$ if $K \cap \bigcap_{i=0}^{n-1} f^{-1}\left(A_{x_{i}}\right) \neq \phi$.

Denote by $S_{n}((\alpha, M), K)$ the largest cardinality of any separated set $E$ of admissible sequences of length $n$ attached to $K$, and put

$$
S_{f}((\alpha, M), K)=\limsup _{n \rightarrow \infty}(1 / n) \log S_{n}((\alpha, M), K) .
$$

Property 1. Under the notations and the assumptions, $S_{f}((\alpha, M), K)$ $\leqq h(f, K)$.

Proof. For $x \in X$ we put 


$$
O(x)=\bigcap\left\{X \backslash A_{i}, x \notin A_{i} . A_{i} \in \alpha\right\} .
$$

Then $\beta=\{O(x) ; x \in X\}$ is an open covering of $X$. For fixed $n>0$, let $E_{n}$ be a separated set of admissible sequences of length $n$ attached to $K$ with the maximal cardinality for $(\alpha, M)$. Let $\gamma$ be a covering of $K$ such that $\gamma \subset \beta_{f}^{n}$ and $K \subset \bigcup_{C \in \gamma} C$. Then for each $\tilde{x}^{*}=\left(x_{0}, \cdots, x_{n-1}\right) \in E_{n}$ there is a $C \in \gamma$ such that $\tilde{x}^{*}$ is attached to $C$. It is easy to see that $\tilde{x}^{*}$ is only one point of $E_{n}$ attached to the $C$. Indeed, write $C=\bigcap_{i=0}^{n-1} f^{-i}\left(O_{i}\right)$ for some $O_{i} \in \beta(0 \leqq i \leqq n-1)$. If $\tilde{y}^{*}=\left(y_{0}, \cdots, y_{n-1}\right) \in E_{n}$ is attached to $C$, then $A_{x_{i}}$ $\cap O_{i} \neq \phi$ and $A_{y_{i}} \cap O_{i} \neq \phi$ for all $0 \leqq i \leqq n-1$. So we have $x^{*}=y^{*}$.

Therefore

$$
S_{n}((\alpha, M), K)={ }^{\sharp} E \leqq N\left(\beta_{f}^{n}\right)
$$

and so

$$
S_{f}((\alpha, M), K) \leqq h(f, K, \beta) \leqq h(f, K) \text {. }
$$

Let $\gamma$ be an open covering of $X$. For $C \in \gamma$ we define

$$
\begin{aligned}
& \operatorname{st}(C)=\bigcup\left\{C^{\prime} \in \gamma ; C \cap C^{\prime} \neq \phi\right\}, \\
& \operatorname{st}(\gamma)=\{\operatorname{st}(C) ; C \in \gamma\} .
\end{aligned}
$$

Property 2. Let $\alpha$ be a finite open covering of $X$ and $\beta$ be a covering of $X$. If $(\beta, N)$ is a CM-pair for $f$ such that $\operatorname{st}(\beta)$ refines $\alpha$, then for $n \geqq 1$

$$
N_{K}\left(\alpha_{f}^{n}\right) \leqq S_{n}((\beta, N), K) \quad \text { and } \quad h(f, K, \alpha) \leqq S_{f}((\beta, N), K)
$$

Proof. Let $E_{n}$ be a set of admissible sequences of length $n$ for $(\beta, N)$. Suppose that $E_{n}$ is separated and attached to $K$ and further has the maximal cardinality. Let $x \in K$. Then there is an admissible sequence $x^{*}=\left(x_{0}, \cdots, x_{n-1}\right)$ such that $x \in \bigcap_{\imath=0}^{n-1} f^{-i}\left(B_{x_{i}}\right)$. Obviously $K \cap \bigcap_{i=0}^{n-1} f^{-i}\left(B_{x_{i}}\right)$ $\neq \phi$. Since $E_{n}$ is maximal, there is $y^{*}=\left(y_{0}, \cdots, y_{n-1}\right) \in E_{n}$ such that $B_{x_{i}}$ $\cap B_{y_{i}} \neq \phi$ for $0 \leqq i \leqq n-1$, and so $x \in \bigcap_{i=0}^{n-1} \operatorname{st}\left(B_{y_{i}}\right)$. This implies that $\left\{\bigcap_{i=0}^{n-1} \operatorname{st}\left(B_{y_{i}}\right) ; y^{*} \in E_{n}\right\}$ is a covering of $K$. Since each $\bigcap_{i=0}^{n-1}$ st $B_{y_{i}}$ contained in at least one element of $\alpha_{f}^{n}$, we have

$$
N_{K}\left(\alpha_{f}^{n}\right) \leqq{ }^{\ddagger} E_{n}=S_{n}((\beta, N), K) \text { and so } h(f, \alpha, K) \leqq S_{f}((\beta, N), K) .
$$

Property 3. For a finite open covering $\alpha$ there is a finite open covering $\gamma$ such that $\operatorname{st}(\gamma)$ refines $\alpha$.

Proof. Recall that compact Haudorff spaces have the uniform structure. We denote by $N(X)$ the family of all neighborhoods of the diagonal 
subset of $X \times X$. Then there exists $L \in N(X)$ such that for all $x \in X$ there is $A \in \alpha$ such that $\{y \in X ;(x, y) \in L\} \subset A$.

Take an open set $U \in N(X)$ such that $U \circ U \circ U \subset L$ (here $A \circ B=$ $\{(x, z) ;(x, y) \in A,(y, z) \in B\})$. Clearly $U[x]=\{y \in X ;(x, y) \in U\}$ is an open neighborhood of $X$, and $\operatorname{st}(U[x]) \subset(U \circ U \circ U)[x] \subset L[x]$ holds. Hence a finite subcovering of $\{L[x] ; x \in X\}$ is the desired one.

Property 4. Let $\mathscr{F}$ be a family of CM-pairs for $f$. Suppose that for any finite open covering $\alpha$ of $X$, there is a CM-pair $(\beta, N) \in \mathscr{F}$ such that $\beta$ refines $\alpha$. Then for $K$ a subset

$$
h(f, K)=\sup _{\left(\beta, N^{\prime}\right) \in \mathscr{F}} S_{f}((\beta, N), K) .
$$

Proof. By Property 1 it is enough to see that

$$
h(f, K) \leqq \sup _{\left(\beta, N^{N}\right) \in \mathscr{F}} S_{f}((\beta, N), K) .
$$

By Property 3 there is a finite open covering $\gamma$ such that st $(\gamma)$ refines $\alpha$. So we choose $(\beta, N) \in \mathscr{F}$ such that $\beta$ refines $\gamma$ by assumption. Obviously $\operatorname{st}(\beta)$ refines $\alpha$. By Property 2 we have

$$
h(f, K, \alpha) \leqq S_{f}((\beta, N), K) \leqq \sup _{\left(\beta, N^{\prime}\right) \in \mathscr{F}} S_{f}((\beta, N), K)
$$

Since $\alpha$ is arbitrary, we obtain the conclusion.

\section{§2. Proof of Theorem 1}

Let $\alpha$ be a finite open covering of $X$ and put

$$
a=\sup _{y \in Y} \inf _{n>0}(1 / n) \log N_{i-1(y)}\left(\alpha_{f}^{n}\right) .
$$

Take and fix $\varepsilon>0$. For any $y \in Y$ there is $m_{y}>0$ such that

$$
\left(1 / m_{y}\right) \log N_{\pi-1(y)}\left(\alpha_{f}^{m_{y}}\right) \leqq a+\varepsilon
$$

and so

$$
N_{\pi-1(y)}\left(\alpha_{f}^{m_{y}}\right) \leqq e^{m_{y^{(}(a+\varepsilon)}} .
$$

From now on we fix $y \in Y$ and choose $\alpha_{y} \subset \alpha_{f}^{m_{y}}$ such that

$$
\pi^{-1}(y) \subset \bigcup_{A \in \alpha_{y}} A, \quad \# \alpha_{y}=N_{\overline{x-1(y)}}\left(\alpha_{f}^{m y}\right) .
$$

Put $O_{y}=\cup_{A \in \alpha_{y}} A$ and denote by $C(y)$ the family of the closed neighbourhoods of $y$. Then we have 


$$
\left(X \backslash O_{y}\right) \cap\left(\bigcap_{k \in C(y)} \pi^{-1}(K)\right)=\phi .
$$

Since $X$ is compact, there is $K_{y} \in C(y)$ such that $\pi^{-1}\left(K_{y}\right) \subset O_{y}$. If $U_{y}$ is the interior of $K_{y}$, then $\left\{U_{y} ; y \in Y\right\}$ is a covering of $Y$. Hence there is a finite subcovering $\beta=\left\{U_{y_{1}}, \cdots, U_{y_{\ell}}\right\}$ of $Y$. For simplicity we write $m_{k}=m_{y_{k}}$ and $U_{h}=U_{y_{h}}$ for $k=1, \cdots, \ell$. Remark that

$$
N_{\bar{\pi}-1\left(C_{k}\right)}\left(\alpha_{f}^{m_{k}}\right)=N_{\pi^{-1}\left(y_{k}\right)}\left(\alpha_{f}^{m_{k}}\right) \leqq e^{m_{k}(a+\varepsilon)} .
$$

Take and fix $n>0$. Then $B \in \beta_{g}^{n}$ is expressed as $B=\bigcap_{i=0}^{n-1} g^{-i}(B(i))$ for some $B(i) \in \beta(0 \leqq i \leqq n-1)$. We fix this $B$ and define recursively a finite sequence $\left\{i_{s}\right\}$ such that

$$
i_{0}=0, \quad i_{s+1}=i_{s}+m_{k} \quad \text { when } B\left(i_{s}\right)=U_{k} .
$$

Let $q$ be the least integer such that $i_{q+1} \geqq n$ and put $n_{s}=m_{k}$ if $B\left(i_{s}\right)=U_{k}$ for $0 \leqq s \leqq q$. Since for any $x \in \pi^{-1}(B)$ and $s$ with $0 \leqq s \leqq q, f^{i s}(x) \in$ $\pi^{-1}\left(B_{i s}\right)$, we have

$$
N_{--1(B)}\left(\alpha_{f}^{u}\right) \leqq \prod_{s=0}^{4} N_{\pi-1\left(B\left(i_{s}\right)\right)}\left(\alpha_{f}^{n s}\right)
$$

and hence

$$
\begin{aligned}
\log N_{n-1(B)}\left(\alpha_{f}^{n}\right) & \leqq \sum_{s=0}^{q} \log N_{\bar{\pi}-\mathbf{1}\left(B\left(i_{s}\right)\right)}\left(\alpha_{f}^{n / s}\right) \\
& \leqq \sum_{s=0}^{q} \log e^{n_{\boldsymbol{s}}(a+\varepsilon)} \\
& \leqq \sum_{s=0}^{q} n_{s}(a+\varepsilon) \\
& \leqq(n+H)(a+\varepsilon)
\end{aligned}
$$

where $H=\max \left\{n_{1}, \cdots, n_{\ell}\right\}$. Therefore

$$
N_{\pi-1(B)}\left(\alpha_{f}^{n}\right) \leqq e^{(n+H)(a+\varepsilon)} .
$$

Since $B$ is arbitrary in $\beta_{g}^{n}$, we have

$$
N\left(\alpha_{f}^{n}\right) \leqq N\left(\beta_{f}^{n}\right) e^{(n+H)(a+\varepsilon)}
$$

and so $h(f, \alpha) \leqq h(g, \varepsilon)+a+\varepsilon \leqq h(g)+a+\varepsilon$. Since $\alpha$ and $\varepsilon$ are arbitrary, the conclusion is obtained.

Remark. We give an example such that the equality of Theorem 1 does not holds. Let $X$ and $f$ be as in Theorem 1 and $Z$ be a compact space. Suppose that $f$ has a fixed point $\left(f\left(x_{0}\right)=x_{0}\right), h(f)>0$ and further 
$(Z, \varphi)$ is a dynamical system such that $h(\varphi)>0$.

Now we put $Y=X \cup Z$ (disjoint union) and define a continuous map $g: Y \rightarrow Y$ by

$$
g(y)= \begin{cases}f(y) & \text { when } y \in X \\ \varphi(y) & \text { when } y \in Z\end{cases}
$$

Set $\pi: Y \rightarrow X$ by

$$
\therefore(y)= \begin{cases}y & \text { when } y \in X \\ x_{0} & \text { when } y \in Z .\end{cases}
$$

Then $h(g)=\max \{h(f), h(\phi)\}$ (see R. L. Adler, A. G. Konheim and M. H. McAndrew [1]). And $\sup _{x \in X} h\left(g, \pi^{-1}(x)\right)=h(\varphi)>0$. So that $h(g)<h(f)$ $+h(\varphi)$.

Proof of Corollary 2. Since $f^{\prime}$ is a factor of $f$, we have $h\left(f^{\prime}\right) \leqq h(f)$. Hence it is enough to show that $h\left(f, \pi^{-1}(x)\right)=0$ for all $x \in X$.

Let $\beta$ be a finite open covering of $X$ such that for all $B \in \beta$ there is a coordinate neighborhood $U_{B}$ with $\operatorname{cl}(B) \subset U_{B}$ (here $\operatorname{cl}(B)$ denotes the closure of $B$ in $X)$. For any $B \in \beta$ let $\xi_{B}$ denote the coordinate map

$$
\xi_{B}: U_{B} \times F \longrightarrow \pi^{-1}\left(U_{B}\right)
$$

where $F$ is the fiber space. Then for fixed $x \in \operatorname{cl}(B)$ the map

$$
\xi_{B, x}: F \longrightarrow E
$$

is defined by $\xi_{B, x}(y)=\xi_{B}(x, y)$ for $y \in F$.

Now take and fix a finite open covering $\alpha$ of $E$. Firstly we show that there is a finite open covering $\gamma_{B}$ of $F$ such that $\gamma_{B}$ refines $\xi_{B, x}^{-1}(\alpha)$ for all $x \in B$. For any $(x, y) \in \operatorname{cl}(B) \times F$ we can find an open neighborhood $U_{y}(x) \times U_{x}(y)$ of $(x, y)$ that is contained in some element of $\xi_{B}^{-1}(\alpha)$. If $y \in F$ is fixed, then $\operatorname{cl}(B) \subset \bigcup_{i=1}^{t} U_{y}\left(x_{i}\right)$ for some finite set $\left\{x_{1}, \cdots, x_{t}\right\}$. So we put $W_{y}=\bigcap_{i=1}^{t} U_{x_{i}}(y)$. Since $\left\{W_{y} ; y \in F\right\}$ is a covering of $F$, we have a finite subcovering $\gamma_{B}=\left\{W_{B}\right\}$ which is the desired one.

We put $r=\left\{\bigcap_{B \in \beta} W_{B} ; W_{B} \in \gamma_{B}\right\}$ and fix $x \in X$. Then for $j \geqq 0$ there is $B(j) \in \beta$ such that $f^{\prime}(x) \in B(j)$, and so put

$$
g_{,}=\left(\xi_{B(j), f \nu\langle x\rangle \mid \pi^{-1}\left(j^{j}, r^{r}\right)}\right)^{-1} \circ f^{j} \circ \xi_{B(j), x} .
$$

Then $g_{j}: F \rightarrow F$ coincides with action of some element of $G$ on $F$. Since $G$ is compact by assumption, the action of $G$ is equicontinuous. Therefore 
there exists an open covering $\gamma^{\prime}$ of $F$ such that $g_{j}^{-1}(\gamma)$ is refined by $\gamma^{\prime}$ for $j \geqq 0$.

Therefore we have $N_{\pi-1(x)}\left(\alpha_{f}^{j}\right) \leqq N_{F}\left(\gamma^{\prime}\right)$ for all $j \geqq 0$, which implies $h\left(f, \pi^{-1}(x), \alpha\right)=0$. Since $\alpha$ is arbitrary, the conclusion is obtained.

\section{§3. Proof of Theorem 3}

Since $\sigma: \Sigma \rightarrow \Sigma$ is the subshift of finite type, there exist a $p \times p$ matrix $M$ of 0 's and 1's and a finite set $S=\{1,2, \cdots, p\}$ such that

$$
\Sigma=\left\{x=\left(x_{i}\right) \in \prod_{j=-\infty}^{\infty} S(j) ; M_{x_{i} x_{i+1}}=1(i \in Z)\right\} .
$$

Here each $S(j)$ denotes the copy of $S$, i.e. $S(j)=S$ for $j \in Z$. Note that the shift $\sigma$ is defined by $\sigma(x)_{i}=x_{i+1}$ for $i \in Z$.

It is well known (cf. $(1,3),[6])$ that $\sigma$ is topologically mixing if and only if $M^{n}>0$ (i.e. $M_{i j}^{n}>0$ for all $i, j$ ) for sufficiently large $n$.

Now we give the proof of Theorem 3 . Let $\ell \geqq 0$. For any

$$
C=\left(a_{-\ell}, \cdots, a_{\ell}\right) \in \prod_{i=-\ell}^{\ell} S(i)
$$

we write

$$
\begin{gathered}
{[C]_{-\ell}=\left\{x \in \Sigma ; x_{i}=a_{i},|i| \leqq \ell\right\},} \\
{[M]_{\ell}=\left\{\left(x_{-\ell}, \cdots, x_{\ell}\right) \in \prod_{i=-\ell}^{\ell} S(i) ; M_{x_{i} x_{i+1}}=1,-\ell \leqq i<\ell\right\} .}
\end{gathered}
$$

Since $[M]_{\ell}$ is finite, we put $[M]_{\ell}=\left\{C^{1}, \cdots, C^{t}\right\}$. If $B_{j}=\pi\left(\left[C^{j}\right]_{-\ell}\right)$ for $1 \leqq$ $j \leqq t$, then $\beta=\left\{B_{1}, \cdots, B_{t}\right\}$ is the family of closed subsets of $X$ and $\beta$ covers $X$.

By using this $\beta$, we construct a CM-pair for $f$. Define a $t \times t$-matrix $B=\left(B_{i j}\right)$ as follows.

In case $\ell=0$, we put

$$
B_{i j}= \begin{cases}1 & \text { when } M_{a_{0}^{i} a_{0}^{j}}=1 \text { where } C^{i}=\left(a_{0}^{i}\right) \text { and } C^{j}=\left(a_{0}^{j}\right), \\ 0 & \text { otherwise }\end{cases}
$$

and in case $\ell>0$

$$
B_{i j}=\left\{\begin{array}{cc}
1 & \text { when } a_{n+1}^{i}=a_{n}^{j}(-\ell \leqq n<\ell) \text { where } \\
& C^{i}=\left(a_{-\ell}^{i}, \cdots, a_{\ell}^{i}\right) \text { and } C^{j}=\left(a_{-\ell}^{j}, \cdots, a_{\ell}^{j}\right) \\
0 & \text { otherwise } .
\end{array}\right.
$$


It is easy to see that $(\beta, B)$ is a CM-pair for $f$.

We denote by $\left(\Sigma_{B}, \sigma_{B}\right)$ the subshift of finite type defined by the matrix $B$ and a finite set $\{1,2, \cdots, t\}$. Since $\sigma: \Sigma \rightarrow \Sigma$ is topologically mixing, there is $m>0$ such that $M^{m}>0$. Then we have $B^{L}>0$ where $L=2 \ell$ $+m$. Hence $\sigma_{B}: \Sigma_{B} \rightarrow \Sigma_{B}$ is topologically mixing.

Let $x \in \Sigma$. For any $i \in Z$ there is a unique $C^{j_{i}} \in[M]_{\ell}$ such that

$$
C^{j i}=\left(x_{i-\ell}, \cdots, x_{i+\ell}\right) \text {. }
$$

So we put $\rho(x)_{i}=j_{i}(i \in Z)$ and $\rho(x)=\left(\rho(x)_{i}\right)$. It is easily checked then that $\rho: \Sigma \rightarrow \Sigma_{B}$ is a homeomorphism and the diagram

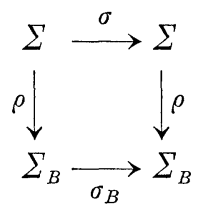

commutes. Define $\pi^{\prime}=\pi \circ \rho^{-1}$. Then $\pi^{\prime}: \Sigma_{B} \rightarrow X$ is surjective and for $1 \leqq$ $j \leqq t$

$$
\begin{aligned}
\pi^{\prime}\left([j]_{0}\right) & =\pi\left(\left\{x \in \Sigma ; \rho(x)_{0}=j\right\}\right) \\
& =\pi\left(\left\{x \in \Sigma ; C^{j}=\left(x_{-\ell}, \cdots, x_{\ell}\right)\right\}\right) \\
& =\pi\left(\left[C^{j}\right]_{-\ell}\right) \\
& =B_{j} .
\end{aligned}
$$

For fixed $n \geqq 1$, let $E_{n}$ be a separated set of admissible sequences attached to $X$ for $(\beta, B)$. We may assume that $E_{n}$ is chosen such that ${ }^{\#} E_{n}$ is maximal. Since $\sigma_{B}: \Sigma_{B} \rightarrow \Sigma_{B}$ is topologically mixing, for $x^{*}=\left(x_{0}, \cdots, x_{n-1}\right)$ $\in E_{n}$ we can find an admissible sequence $\tilde{x}^{*}=\left(x_{0}, \cdots, x_{L+n-1}\right)$ such that $x_{0}=x_{L+n-1}$. Put $\tilde{E}_{n}=\left\{\tilde{x}^{*} ; x^{*} \in E_{n}\right\}$. Since $E_{n}$ is separated, so is $\tilde{E}_{n}$. For any $\tilde{y}^{*} \in \tilde{E}_{n}$ define a periodic point $y=(y)_{i \in Z}$ by

$$
y_{i}=y_{r} \quad(i=(L+n-1) p+r, 0 \leqq r<L+n-1, p \in Z) .
$$

Obviously $y$ has the period $L+n-1$ and $y \in \Sigma_{B}$. For $\tilde{y}^{*}, \tilde{z}^{*} \in \tilde{E}_{n}$, as above there exist periodic points $y, z \in \Sigma_{B}$ corresponding to $\tilde{y}^{*}, \tilde{z}^{*}$ repsectively. If $\tilde{y}^{*} \neq \tilde{z}^{*}$, then we have $\pi^{\prime}(y) \neq \pi^{\prime}(z)$ since $\left\{\tilde{y}^{*}, \tilde{z}^{*}\right\}$ is separated. Hence we have

$$
{ }^{\sharp} E_{n}={ }^{\sharp} \tilde{E}_{n} \leqq N_{K+n-1}(f),
$$

i.e. $S_{n}((\beta, B), X) \leqq N_{L+n-1}(f)$ since ${ }^{\sharp} E_{n}$ is maximal. 
Now let $\alpha$ be a finite open covering of $X$. By the construction of $\beta$ we have that $\operatorname{diam}(\beta) \rightarrow 0$ when $\ell \rightarrow \infty$ (here $\operatorname{diam}(\beta)=\max \left\{\operatorname{diam}\left(B_{i}\right)\right.$; $1 \leqq i \leqq t\}$ ). Hence there is $\beta$ such that st $(\beta)$ refines $\alpha$. From Property 2 and the above inequality we have

$$
\begin{aligned}
h(f, \alpha) & =\lim _{n \rightarrow \infty}(1 / n) \log N\left(\alpha_{f}^{n}\right) \\
& \leqq \liminf _{n \rightarrow \infty}(1 / n) \log S_{n}((\beta, N), X) \\
& \leqq \liminf _{n \rightarrow \infty}(1 / n) \log N_{L+n-1}(f) \\
& \leqq \liminf _{n \rightarrow \infty}(1 / n) \log N_{n}(f),
\end{aligned}
$$

and therefore the desired inequality is obtained.

Remark. Under the notations and the assumptions of Theorem 3, we can construct an example such that

$$
h(f) \varsubsetneqq \liminf _{n}(1 / n) \log N_{n}(f) .
$$

Let $\Sigma$ be a shift space defined by two symbols $\{0,1\}$ and as before define a shift $\sigma: \Sigma \rightarrow \Sigma$. For $k \in Z(k>0)$ we consider a point $p^{k}=\left(p_{i}^{k}\right)$ $\in \Sigma$ defined by

$$
p_{i}^{k}= \begin{cases}1 & \text { when }[i / k] \text { is even } \\ 0 & \text { otherwise }\end{cases}
$$

where [ ] denotes the Gauss' symbol. Obviously each $p^{k}$ is a periodic point of period $2 k$. For this point $p^{k}$ we denote by $O_{k}(p)$ the orbit of $\sigma$.

Consider two the matrices $A=\left(\begin{array}{ll}1 & 0 \\ 1 & 1\end{array}\right)$ and $B=\left(\begin{array}{ll}1 & 1 \\ 0 & 1\end{array}\right)$ and define shift spaces of finite type which are denoted by $\Sigma_{A}$ and $\Sigma_{B}$. Let $p^{\omega}$ be a point in $O_{\omega}=\Sigma_{A} \cup \Sigma_{B}$. We define a new space $X$ by

$$
X=\left(\Sigma-\left(O_{1}(p) \cup O_{2}(p) \cup \cdots \cup O_{\omega}\right)\right) \cup\left\{p^{1}, p^{2}, \cdots, p^{\omega}\right\}
$$

and construct a map $\pi: \Sigma \rightarrow X$ by

$$
\pi(x)= \begin{cases}p^{k} & \text { when } x \in O_{k}(p) \quad(k=1,2, \cdots, \omega) \\ x & \text { otherwise }\end{cases}
$$

Obviously $\pi$ is surjective. So we introduce the strongest topology in $X$ for which $\pi$ is continuous. It is easily checked then that $X$ is Hausdorff and compact. Hence the product topological space $\Sigma \times X$ is Hausdorff and compact. 
It is easy to see that a continuous map $f: X \rightarrow X$ is induced from $\sigma$ by $\pi$. Then $f$ has infinitely many fixed points. In fact, $f\left(p^{k}\right)=p^{k}$ for $k=1,2, \cdots, \omega$. Put $\rho(x, y)=(x, \pi(y))$ for $(x, y) \in \Sigma \times \Sigma$. Then the diagram

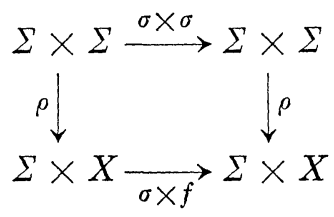

commutes. $(\Sigma \times \Sigma, \sigma \times \sigma)$ is a subshift of finite type and topologically mixing, and $h(\sigma \times f) \leqq h(\sigma \times \sigma)=2 \log 2$ holds. However $N_{n}(\sigma \times f)$ is infinite for $n>0$.

\section{REFERENCES}

[1] R. L. Adler, A. G. Konheim and M. H. McAndrew, Topological entropy, Trans. Amer. Math. Soc., 114 (1965), 309-319.

[2] R. Bowen, Topological entropy and Axiom A, Global analysis, Proc. Sympos. Pure Math., 14 (1970), AMS, 23-42.

[3] _ Periodic points and measures for Axiom $A$ diffeomorphisms, Trans. Amer. Math. Soc., 154 (1971), 377-397.

[4] _ Entropy for group endomorphisms and homogeneous spaces, Trans. Amer. Math. Soc., 153 (1971), 401-414, 181 (1973), 509-510.

[ 5 ] - Erratume to "Entropy for group endomorphisms and homogeneous spaces", Trans. Amer. Math. Soc., 181 (1973), 509-510.

[6] - Equilibrium states and the ergodic theory of Anosov diffeomorphisms, Lecture Notes in Math., 470 (1975), Springer Verlag.

[ 7 ] J. L. Kelley, General topology, University series in higher mathematics, Van Nostrand, Toronto, New York, London (1955).

[8] P. Walters, Ergodic Theory-Inductory Lectures, Lecture Notes in Math., 458 (1975), Springer Verlag.

Department of Mathematics

Faculty of Science

Nagoya University

Chikusa-ku, Nagoya 464

Japan 